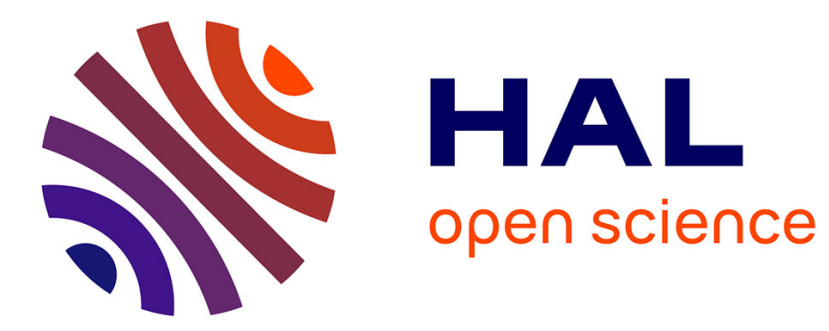

\title{
Mechanical derivation of elastic constants of $\epsilon$-martensite
} Thiebaud Richeton

\section{To cite this version:}

Thiebaud Richeton. Mechanical derivation of elastic constants of $\epsilon$-martensite. Scripta Materialia, 2019, 169, pp.14-18. 10.1016/j.scriptamat.2019.04.042 . hal-02195952v2

\section{HAL Id: hal-02195952 https://hal.univ-lorraine.fr/hal-02195952v2}

Submitted on 26 Aug 2019

HAL is a multi-disciplinary open access archive for the deposit and dissemination of scientific research documents, whether they are published or not. The documents may come from teaching and research institutions in France or abroad, or from public or private research centers.
L'archive ouverte pluridisciplinaire HAL, est destinée au dépôt et à la diffusion de documents scientifiques de niveau recherche, publiés ou non, émanant des établissements d'enseignement et de recherche français ou étrangers, des laboratoires publics ou privés. 


\title{
Mechanical derivation of elastic constants of $\varepsilon$-martensite ${ }^{1}$
}

\author{
T. Richeton ${ }^{2}$ \\ Université de Lorraine, CNRS, Arts et Métiers ParisTech, LEM3, F-57000 \\ Metz, France
}

\begin{abstract}
This work presents direct formulas to compute the HCP elastic constants of $\varepsilon$-martensite from the FCC ones of austenite. The derivation is performed thanks to mechanical compatibility arguments while considering $\varepsilon$-martensite as a laminate structure of twinned and untwinned FCC phases. The formulas are applied to predict the elastic constants of several alloys and very good agreement with measurements is found. According to these relations, directional Young moduli of $\varepsilon$-martensite are most of the time stiffer than the austenite ones and the (0001) shear modulus is always greater than the (111) one, which hardens dislocation glide parallel to the interfaces.
\end{abstract}

Keywords: martensitic phase transformation, elastic behavior, micromechanical modeling, austenitic steels, anisotropic elasticity

The martensitic transformation $\gamma(\mathrm{FCC}) \longrightarrow \varepsilon(\mathrm{HCP})$ may occur in several crystalline materials during deformation or cooling. It can be observed in low stacking fault energy austenitic steels $\left[\mathrm{GPH} 05, \mathrm{IRV}^{+} 09, \mathrm{BAS}^{+} 11\right]$, in Febased shape memory alloys [ST74, YW92, BGE97, $\left.\mathrm{NBL}^{+} 10\right]$ or in Co alloys [FD67, WG75, KHRN18] for instance. The part of $\varepsilon$-martensite can be significant [GPH05], and the size and the distribution of martensitic constituents are primordial issues for the material in-use properties.

Elastic constants of $\varepsilon$-martensite are generally badly known for most materials, in particular in high manganese austenitic steels $\left[\mathrm{BAS}^{+} 11, \mathrm{GMK}^{+} 11\right.$, $\left.\mathrm{PNM}^{+} 13\right]$. Due to its lower ductility, a good understanding of the elastic behavior of $\varepsilon$-martensitic phase is actually even more important. The difficulty to measure accurately anisotropic elastic constants of a specific phase of an alloy

\footnotetext{
${ }^{1}$ Scripta Materialia 169 (2019) 14 - 18, https://doi.org/10.1016/j.scriptamat.2019.04.042

${ }^{2}$ Corresponding author. Tel.:+33 3727478 02, thiebaud.richeton@univ-lorraine.fr
} 
arises typically from the impossibility to produce a single crystal with exactly the same composition. On the other hand, direct measurements on multiphase alloys involve the use of inverse methods, such as the self-consistent scheme, and suffer from the non-uniqueness of the solutions. However, the knowledge of elastic constants may be of prime importance for mechanical modeling of internal stresses through mean-field elasto-(visco)plastic approaches (e.g. $\left.\left[\mathrm{LBG}^{+} 18\right]\right)$, full-field crystal plasticity simulations (e.g. $\left.\left[\mathrm{REH}^{+} 10\right]\right)$ or investigations at the dislocation scale like evaluation of image forces on dislocations due to elastic mismatch between phases [PK94, JD18, CRCS19]. Furthermore, anisotropic elastic constants are also needed if one wants to estimate accurately local stresses from elastic strain measurements by diffraction (e.g. [KMRM94, WMD06, BBP ${ }^{+}$14]). In particular, the load transfers between austenite and $\varepsilon$-martensite, generally stronger and harder $\left[\mathrm{NBL}^{+} 10, \mathrm{BAS}^{+} 11\right]$, depend closely on the values of the elastic stiffnesses of each phase. The correct estimation of these load transfers is especially crucial to control damage resistance in steels.

The martensitic transformation $\gamma(\mathrm{FCC}) \longrightarrow \varepsilon(\mathrm{HCP})$ is reported to be due to the glide of $a / 6\langle 11 \overline{2}\rangle$ Shockley partial dislocations on every second closepacked $(111)_{\gamma}$ plane, which corresponds to the introduction of a stacking fault in each second $(111)_{\gamma}$ plane (see Figure 1a) $\left[\mathrm{IRV}^{+} 09\right]$. As a result, the $\varepsilon$ martensitic phase has the following crystallographic relationship with the $\gamma$ phase:

$$
(111)_{\gamma}\left\|(0001)_{\varepsilon},\langle 11 \overline{2}\rangle_{\gamma}\right\|\langle 01 \overline{1} 0\rangle_{\varepsilon},\langle 1 \overline{1} 0\rangle_{\gamma} \|\langle 2 \overline{1} \overline{1} 0\rangle_{\varepsilon}
$$

which is known as the Shoji-Nishiyama orientation relationship [Nis78]. This process is actually very similar to the mechanism of $\Sigma 3(111)$ twin formation in FCC crystals which also occurs by glide of Shockley partials but on each successive $(111)_{\gamma}$ plane, resulting in the introduction of a stacking fault in each $(111)_{\gamma}$ plane (see Figure 1b). In Figure 1, it is seen that the crossing from an untwinned to a twinned FCC crystal is described by a sequence $A B A$ which is also present in the HCP stacking sequence. As a consequence, it may be possible to consider the HCP $\varepsilon$-martensitic phase as a periodic laminate structure made of alternating twinned and untwinned FCC phases with equal volume fractions as pictured in Figure 2. Accordingly, the idea of the present work is to compute the elasticity tensor of $\varepsilon$-martensite as the effective elasticity tensor of a laminate composed of alternating twinned and untwinned FCC phases.

To perform this derivation, let first consider a general infinite periodic lam- 


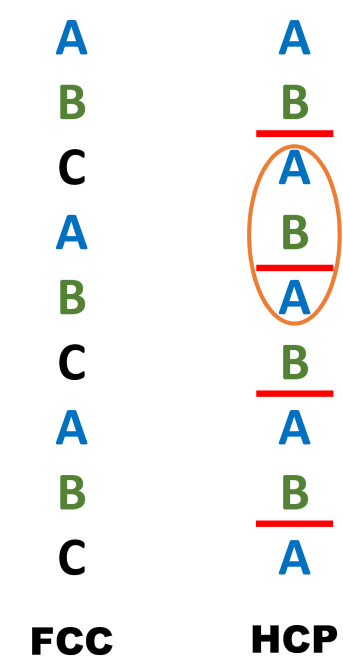

(a)

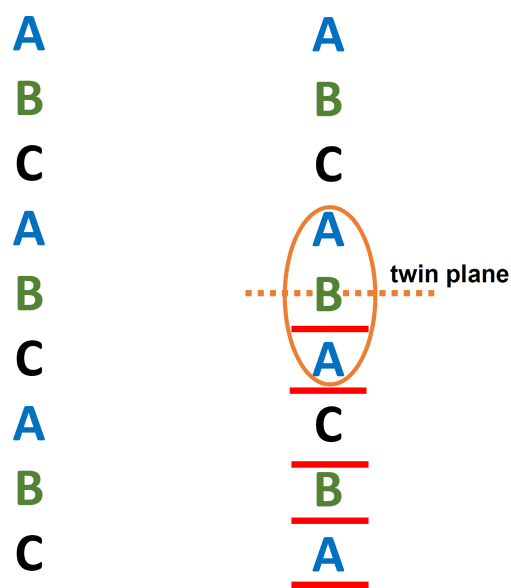

FCC

(b)

Figure 1: (a) FCC and HCP stacking sequences. (b) FCC stacking sequences for a perfect crystal and one with a twin plane (mirror symmetry). The red lines represent stacking faults in the FCC sequence. The common sequence $A B A$ of the HCP and parent/twin structures is surrounded in orange.

inate structure composed of two alternating crystals, $I$ and $I I$, in a Cartesian orthonormal frame $\left(\underline{e_{1}}, \underline{e_{2}}, \underline{e_{3}}\right)$ (Figure 2$)$. The crystals are assumed perfectly bonded along the interfaces oriented by their unit normal $\underline{n} \equiv \underline{e_{3}}$. The laminate structure is supposed to have a pure linear elastic behavior in response to a macroscopic homogeneous and remotely applied stress $\Sigma_{i j}$. The volume fractions of crystals $I$ and $I I$ are denoted $f^{I}$ and $f^{I I}$, respectively. Hereafter, the superscript $I$ denotes fields in crystals $I$ and the superscript $I I$ fields in crystals II.
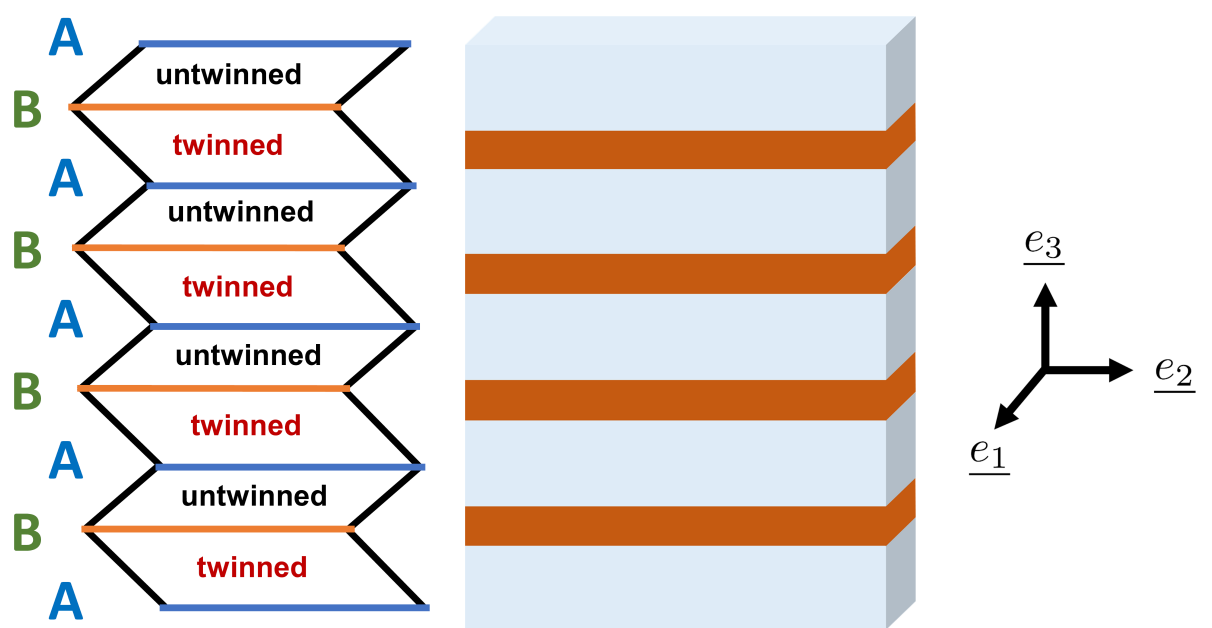

Figure 2: HCP stacking sequence seen as a periodic alternating structure of twinned and untwinned FCC phases and model of an infinite periodic laminate structure composed of two alternating crystals with different volume fractions. 
Through consideration of strains compatibility and balance of linear momentum without body force, it can be shown that the Cauchy stresses $\sigma_{i}^{I, I I}$ are uniform in crystals $I$ and $I I$ and that their expressions can be analytically derived [GPK89, RB13]. Denoting $s_{i j}$ the components of the elastic compliance tensor and using the contracted Voigt notation [Voi28] $(11 \rightarrow 1,22 \rightarrow 2,33 \rightarrow$ $3,23 \rightarrow 4,31 \rightarrow 5,12 \rightarrow 6)$ along with the notations $\llbracket s_{i j} \rrbracket=s_{i j}^{I I}-s_{i j}^{I}$ and $\tilde{s}_{i j}=$ $f^{I I} s_{i j}^{I}+f^{I} s_{i j}^{I I}$, they may be written as [RB13, RTBB15, $\mathrm{TRM}^{+} 15$, Ric17]:

$$
\begin{gathered}
\sigma_{i}^{I}=\Sigma_{i}+f^{I I} G_{i k} \llbracket s_{k j} \rrbracket \Sigma_{j} \\
\sigma_{i}^{I I}=\Sigma_{i}-f^{I} G_{i k} \llbracket s_{k j} \rrbracket \Sigma_{j}
\end{gathered}
$$

where the Einstein summation convention over repeated indices is used, indices range from 1 to 6 and the non-zero components of the symmetric tensor $G_{i j}$ read:

$$
\begin{gathered}
G_{11}=\left(\tilde{s}_{26}^{2}-\tilde{s}_{22} \tilde{s}_{66}\right) / D, \quad G_{12}=\left(\tilde{s}_{12} \tilde{s}_{66}-\tilde{s}_{16} \tilde{s}_{26}\right) / D, \\
G_{22}=\left(\tilde{s}_{16}^{2}-\tilde{s}_{11} \tilde{s}_{66}\right) / D, \quad G_{16}=\left(\tilde{s}_{22} \tilde{s}_{16}-\tilde{s}_{12} \tilde{s}_{26}\right) / D, \\
G_{66}=\left(\tilde{s}_{12}^{2}-\tilde{s}_{11} \tilde{s}_{22}\right) / D, \quad G_{26}=\left(\tilde{s}_{11} \tilde{s}_{26}-\tilde{s}_{12} \tilde{s}_{16}\right) / D, \\
\text { with } D=\tilde{s}_{22} \tilde{s}_{16}^{2}+\tilde{s}_{11} \tilde{s}_{26}^{2}+\tilde{s}_{66} \tilde{s}_{12}^{2}-\tilde{s}_{11} \tilde{s}_{22} \tilde{s}_{66}-2 \tilde{s}_{12} \tilde{s}_{16} \tilde{s}_{26} .
\end{gathered}
$$

By definition, the effective compliance tensor $s_{i j}^{e f f}$ of the laminate structure satisfies $\left\langle\varepsilon_{i}\right\rangle=s_{i j}^{e f f} \Sigma_{j}$ where \langle\rangle denotes a volume average. Hence, from relation 2, we have:

$$
\left\langle\varepsilon_{i}\right\rangle=\left(\left\langle s_{i l}\right\rangle-f^{I} f^{I I} \llbracket s_{i j} \rrbracket G_{j k} \llbracket s_{k l} \rrbracket\right) \Sigma_{l}
$$

and then by identification, the effective compliance tensor may be expressed as:

$$
s_{i j}^{e f f}=\left\langle s_{i j}\right\rangle-f^{I} f^{I I} \llbracket s_{i k} \rrbracket G_{k l} \llbracket s_{l j} \rrbracket .
$$

In order to retrieve the structure of $\varepsilon$-martensite as described in Figure 2, it is thereafter considered that the crystals have a FCC structure and that the interfaces correspond to $\Sigma 3(111)$ twin boundaries with $I$ the parent phase and $I I$ the twin phase. The Zener anisotropy ratio $A$ is then introduced in order to express the elastic compliance tensors of crystals $I$ and $I I, s_{i j}^{I, I I}$, in the frame $\left(\underline{e_{1}}, \underline{e_{2}}, \underline{e_{3}}\right)$ of Figure 2. By definition, $A=2\left(s_{11}^{*}-s_{12}^{*}\right) / s_{44}^{*}$ where $s_{i j}^{*}$ designate the elastic compliances in the orthonormal axis system aligned with respect 
to the $\langle 100\rangle$ directions of the cubic crystal. Without loss of generalization, it is supposed that $\underline{e}_{1}$ is collinear to the twinning shear direction which corresponds to a $\langle 11 \overline{2}\rangle$ crystallographic direction, whereas $e_{3}$ is taken as the normal of (111) planes. Accordingly, $s_{i j}^{I}$ is deduced from $s_{i j}^{*}$ by the appropriate rotation and $s_{i j}^{I I}$ by a $180^{\circ}$ rotation of $s_{i j}^{I}$ around $\frac{e_{3}}{s^{I}}$ [RTBB15]. As a result, the non-zero components of the symmetric tensor $\overline{s_{i j}^{I}}$ can be related to $A$ as follows:

$$
\begin{aligned}
& s_{11}^{I}=s_{22}^{I}=s_{11}^{*}-\frac{A-1}{4} s_{44}^{*} \\
& s_{12}^{I}=s_{12}^{*}+\frac{A-1}{12} s_{44}^{*} \\
& s_{13}^{I}=s_{23}^{I}=s_{12}^{*}+\frac{A-1}{6} s_{44}^{*} \\
& s_{14}^{I}=-s_{24}^{I}=\frac{1}{2} s_{56}^{I}=\frac{\sqrt{2}}{6}(A-1) s_{44}^{*} \\
& s_{33}^{I}=s_{11}^{*}-\frac{A-1}{3} s_{44}^{*} \\
& s_{44}^{I}=\frac{2(A+1)}{3} s_{44}^{*} \\
& s_{66}^{I}=\frac{A+2}{3} s_{44}^{*}
\end{aligned}
$$

whereas $s_{i j}^{I I}$ is identical to $s_{i j}^{I}$, except for the following components which have opposite signs:

$$
s_{14}^{I I}=-s_{24}^{I I}=\frac{1}{2} s_{56}^{I I}=-s_{14}^{I}
$$

Hence, many components of $\llbracket s_{i j} \rrbracket$ and $\left\langle s_{i j}\right\rangle$ are zero, especially in the case of equal volume fractions which corresponds to the description of the $\varepsilon$-martensite structure made in Figure 2 where twinned and untiwnned phases have same volume. In this particular case, $s_{i j}^{e f f}$ can be easily derived from Eq.5:

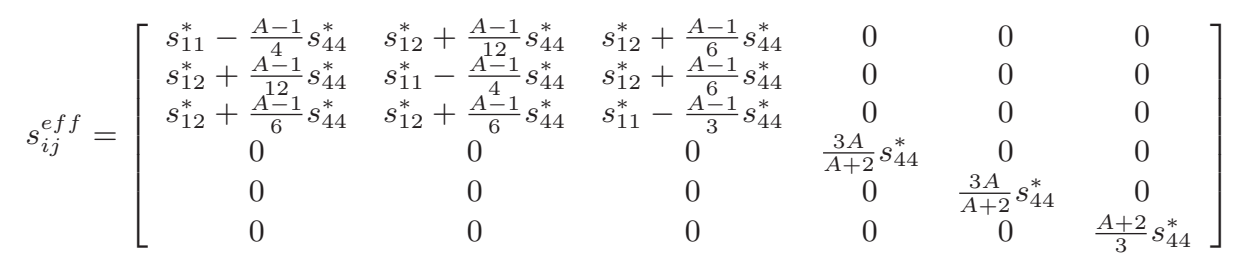

It is observed that $s_{i j}^{e f f}$ is symmetric with respect to a rotation about $\underline{e_{3}}$, i.e. the effective medium is transversely isotropic for equal volume fractions. From the previous developed arguments, it seems relevant to use this transversely 
isotropic tensor to obtain a good estimation of the HCP elastic compliance tensor of $\varepsilon$-martensite, although such a procedure ignores of course the chemical processes at stake during the martensitic transformation, like diffusion of solutes or segregation of chemical elements.

The stiffness tensor is simply the inverse of $s_{i j}^{e f f}$. As a result, very simple and direct formulas that allow to get from the FCC elastic constants of austenite to the five independent HCP elastic constants of $\varepsilon$-martensite are obtained:

$$
\begin{array}{rlrl}
c_{11}^{H C P} & =c_{11}^{*}+\frac{A^{2}+7 A-8}{3 A(A+2)} c_{44}^{*} & c_{12}^{H C P}=c_{12}^{*}+\frac{A^{2}-5 A+4}{3 A(A+2)} c_{44}^{*} \\
c_{13}^{H C P}=c_{12}^{*}-\frac{2(A-1)}{3 A} c_{44}^{*} & c_{33}^{H C P}=c_{11}^{*}+\frac{4(A-1)}{3 A} c_{44}^{*} \\
c_{44}^{H C P}=\frac{A+2}{3 A} c_{44}^{*} &
\end{array}
$$

where $c_{11}^{*}, c_{12}^{*}$ and $c_{44}^{*}$ are taken in the orthonormal axis system aligned with respect to the $\langle 100\rangle$ directions of the cubic crystal.

It is noteworthy that a previous procedure relating the elasticity tensors of HCP and FCC structures already existed. It was developed by Martin [Mar72] for wurtzite (HCP) and zinc-blende (FCC) structure materials and then applied by Fuller and Weston to metallic structures [FW74]. The starting point of their method is the construction of wurtzite and zinc-blende structures from tetrahedral building blocks whose corners lie along $\langle 111\rangle$ directions [Rob68]: for zinc-blende structure, tetrahedra are aligned in equivalent orientations and for wurtzite structure, tetrahedra are alternatively aligned in two mirror orientations [Mar72, FW74] which can be related to the frame $\left(\underline{e_{1}}, \underline{e_{2}}, \underline{e_{3}}\right)$ and the associated twinned phase previously defined. Then, according to the procedure of Martin [Mar72], the elastic constants of the wurtzite structure are deduced from the minimization of its elastic strain energy density $U$ (computed as the average density of the two trigonal orientations) with respect to the difference of strains in the two trigonal orientations $\llbracket \varepsilon_{i} \rrbracket$ :

$$
\frac{\partial U}{\partial \llbracket \varepsilon_{i} \rrbracket}=0
$$

As a result of this minimization, the following transformation formulas are obtained [Mar72, FW74]:

$$
c_{i j}^{H C P}=\left\langle c_{i j}\right\rangle-\llbracket c_{i k} \rrbracket\left\langle c_{k l}\right\rangle^{-1} \llbracket c_{l j} \rrbracket
$$


The first term of the sum in Eq.11 is a simple average of elastic stiffnesses whereas the last term is called an internal strain contribution [Mar72, FW74]. This expression shares some similarities with the one of the effective compliance tensor (Eq.5), which is also composed of a first average term and a second one that depends on differences. It is nevertheless not strictly equivalent. It must be underlined for instance that in general $\left\langle s_{i j}\right\rangle^{-1} \neq\left\langle c_{i j}\right\rangle$. Actually, among the five independent elastic constants, the internal strain contribution of Eq.11 is non-zero for $c_{11}^{H C P}, c_{12}^{H C P}$ and $c_{44}^{H C P}$ whereas the right hand-side member of Eq.5 is non-zero only for $s_{44}^{H C P}$.

It can be easily checked that the results provided by Eq. 11 are the same as the ones given by Eq.9, with the notable exception of $c_{44}^{H C P}$ for which Eq.9 actually translates into $c_{44}^{H C P}=\left\langle c_{44}\right\rangle$. Precisely about $c_{44}^{H C P}$, Fuller and Weston [FW74] noticed that the computed elastic constants are in better agreement with experimental measurements without considering the internal strain contribution and wrote "there is no basis for this except that it gives the correct pattern for the internal strain contributions". It is therefore worth underlying that the present work offers a rigorous mechanical justification and presents the formulas in a very simple form that allows straightforward applications. Furthermore, it is interesting to resume the different comparisons with measured constants performed by Martin [Mar72] and Fuller and Weston [WG75] using Eq.9. Four materials, $\mathrm{ZnS}$, Co-32wt.\% Ni, Co and $\mathrm{Tl}$, exhibiting the $\varepsilon$-martensitic transformation are thus considered in Table 1. HCP elastic constants are computed from Eq.9 considering experimentally measured values for the FCC constants and are compared with experimental values. It must be noticed however that the presented HCP and FCC experimental values refer to the exact same compound only for $\mathrm{ZnS}$ and Co-32wt.\%Ni alloys. For pure cobalt and pure thallium, the $\mathrm{HCP}$ values are extrapolations derived from measurements of alloys of different compositions.

It can be observed that the agreement between the computed and the experimental values is pretty good. In all the cases, the differences between constants are always smaller than $10 \mathrm{GPa}$. For the two most reliable cases, $\mathrm{ZnS}$ and Co$32 w t . \% \mathrm{Ni}$, the relative errors are less than $7 \%$, which can be considered within experimental errors.

From Eq.9, the elastic heterogeneity of a multiphase alloy composed of both $\gamma$-austenite and $\varepsilon$-martensite can be evaluated. Considering for instance typical values of elastic constants for austenitic steels, Fig.3 displays the magnitude of the directional Young modulus of both phases in all the space directions. In 
Table 1: Comparisons between experimental HCP elastic constants of different alloys and the ones computed from Eq.9 considering the following FCC elastic constants: for ZnS, $c_{11}^{*}=103.87, c_{12}^{*}=$ 65.07 and $c_{44}^{*}=46.20$ (average of Refs. [BJS67] and [Zar63]), for Co-32wt.\% Ni, $c_{11}^{*}=238.7$, $c_{12}^{*}=155.3$ and $c_{44}^{*}=131.5$ [WG75], for Co, $c_{11}^{*}=239.8, c_{12}^{*}=163.4$ and $c_{44}^{*}=133.4$ [LW70], for $\mathrm{Tl}, c_{11}^{*}=40.8, c_{12}^{*}=34.0$ and $c_{44}^{*}=11.0$ [SS67]. All data are taken at room temperature. Constant are in $\mathrm{GPa}$ and errors in \%.

\begin{tabular}{llllllllllllll}
\hline & \multicolumn{3}{c}{ ZnS } & \multicolumn{4}{c}{ Co-32wt.\%Ni } & \multicolumn{3}{c}{ Co } & \multicolumn{3}{c}{ Tl } \\
& Exp. & Comp. & Err. & Exp. & Comp. & Err. & Exp. & Comp. & Err. & Exp. & Comp. & Err. \\
\hline$c_{11}$ & 124.2 & 125.0 & 0.6 & 326.0 & 303.5 & 6.9 & 306.3 & 306.2 & 0.0 & 40.8 & 46.2 & 13.2 \\
$c_{33}$ & 140.0 & 139.6 & 0.3 & 358.4 & 358.4 & 0.0 & 357.4 & 366.7 & 2.6 & 52.8 & 50.9 & 3.6 \\
$c_{12}$ & 60.2 & 61.8 & 2.7 & 160.6 & 150.4 & 6.4 & 165.1 & 160.5 & 2.8 & 35.4 & 33.6 & 5.1 \\
$c_{13}$ & 45.5 & 47.2 & 3.7 & - & 95.4 & - & 101.9 & 99.9 & 2.0 & 29.0 & 28.9 & 0.3 \\
$c_{44}$ & 28.6 & 28.3 & 1.0 & 74.0 & 71.6 & 3.2 & 75.3 & 69.9 & 7.1 & 7.3 & 5.9 & 19.2 \\
\hline
\end{tabular}

Fig.3, the anisotropy of the FCC phase is significant with $A=3.36$ but it is seen that it is much less pronounced in the HCP phase. Along the normal to the $\gamma / \varepsilon$ phase boundary which is parallel to $\langle 111\rangle_{\gamma}$ and $\langle 0001\rangle_{\varepsilon}$, the value of the Young modulus is actually maximal and equal with $E_{F C C}^{\max }=E_{H C P}^{\max }=288$ GPa. However, strong differences of Young modulus can be observed along other directions and it was checked that $\varepsilon$-martensite is much stiffer along a set of crystallographic directions representing a predominant part of the orientation space. In particular, the minimal value is much higher in $\varepsilon$-martensite, $E_{H C P}^{\min }=$ $172 \mathrm{GPa}$, than in austenite, $E_{F C C}^{\min }=101 \mathrm{GPa}$. The fact that the Young modulus should be for most directions higher in $\varepsilon$-martensite is consistent with some local nanoindentation measurements in a Fe-based shape memory alloy which showed greater values of Young modulus and hardness in $\varepsilon$-martensitic grains compared to austenitic ones $\left[\mathrm{NBL}^{+} 10\right]$.

Besides, it is noteworthy that $\varepsilon$-martensite variants can form lamellar structures within grains where $\gamma / \varepsilon$ phase boundaries may act as obstacles to dislocation motion [YW92, GPH05, $\left.\mathrm{BAS}^{+} 11\right]$. In such confined environment, dislocation glide parallel to interfaces is promoted due to less slip resistance (e.g. [DDR $\left.\left.{ }^{+} 12\right]\right)$. As dislocation glide is also controlled by the value of the shear modulus of the slip plane, it is therefore worth comparing the values of the shear modulus in the $(111)_{\gamma}$ and $(0001)_{\varepsilon}$ planes. From Eqs.6 and 9, it is deduced that these shear moduli are isotropic within their planes and that they can be expressed as:

$$
\mu_{(111)_{\gamma}}=\frac{3}{2 A+1} c_{44}^{*} \quad \mu_{(0001)_{\varepsilon}}=\frac{A+2}{3 A} c_{44}^{*}
$$

Accordingly, their ratio can be written in an original manner only with re- 


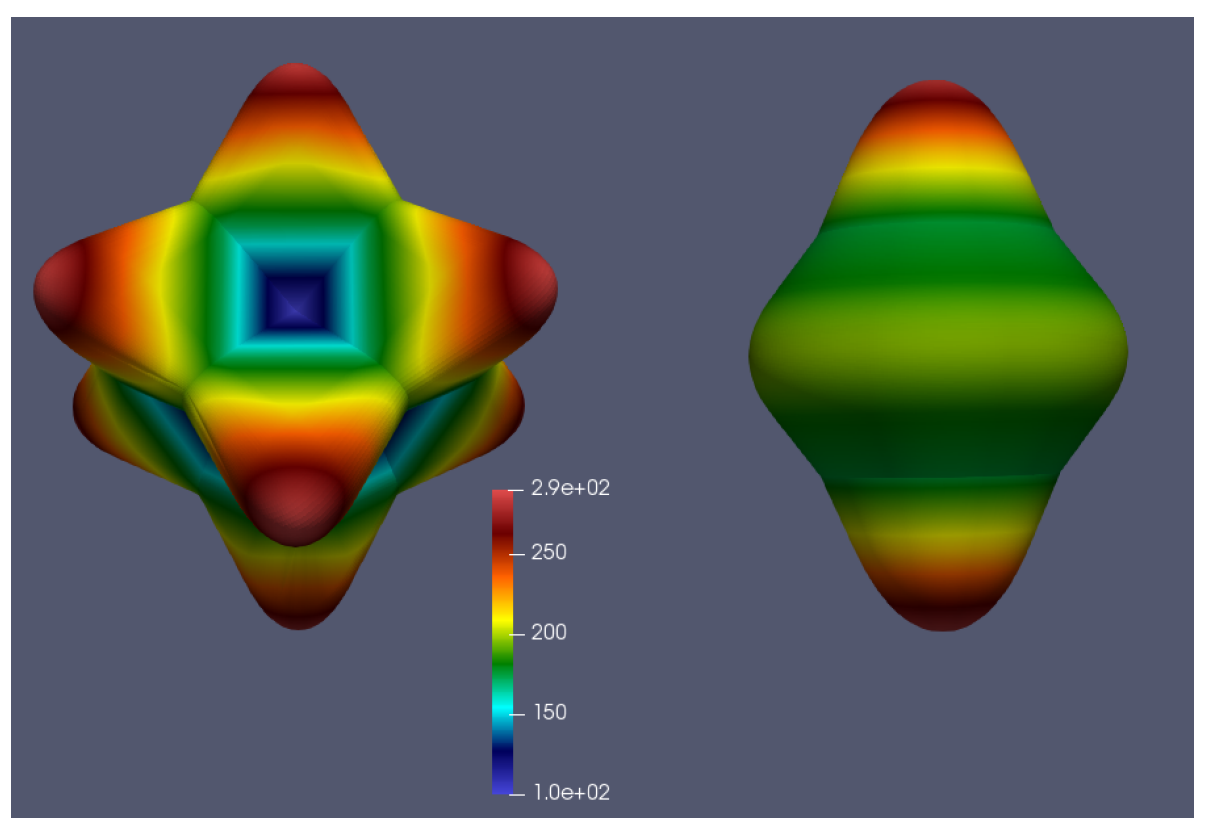

Figure 3: On the left, magnitude of the directional Young modulus of the FCC phase of an austenitic steel in all the space directions considering $c_{11}^{*}=197.5 \mathrm{GPa}, c_{12}^{*}=125 \mathrm{GPa}$ and $c_{44}^{*}=122 \mathrm{GPa}$ [Sau06]. On the right, magnitude of the directional Young modulus of the corresponding HCP phase computed from Eq.9. The vertical axis is aligned with the $\langle 111\rangle_{\gamma}$ and $\langle 0001\rangle_{\varepsilon}$ crystallographic directions, respectively.

spect to $A$ :

$$
\frac{\mu_{(0001)_{\varepsilon}}}{\mu_{(111)_{\gamma}}}=\frac{2}{9}\left(A+\frac{5}{2}+\frac{1}{A}\right)
$$

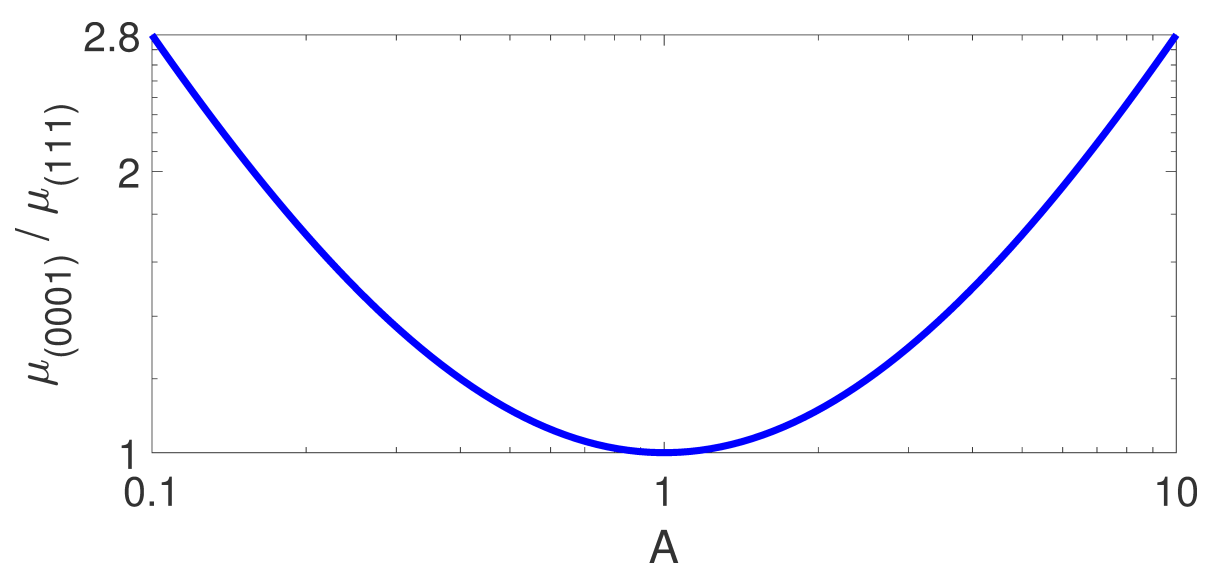

Figure 4: Variation of the shear modulus ratio $\frac{\mu_{(0001)_{\varepsilon}}}{\mu_{(111)_{\gamma}}}$ (Eq.13) with the Zener anisotropy ratio $A$ in $\log -\log$ scale.

Fig. 4 shows then that the variation of this ratio with $A$ is symmetric around 1 in a log-log scale. From Eq.13 and Fig.4, it is noticed that both shear moduli are equal for $A=1$ but that $\mu_{(0001)_{\varepsilon}}$ is always greater than $\mu_{(111)_{\gamma}}$ otherwise. The 
increase of the shear modulus becomes significant as soon as $A$ departs from unity. For instance, an increase of $37 \%$ is obtained for $A=3.36$. Apart from other considerations, this finding indicates that it should be much more difficult to make dislocations glide along $(0001)_{\varepsilon}$ planes than along $(111)_{\gamma}$ ones, which is consistent with the observed pronounced hardening in presence of $\varepsilon$ martensite variants $\left[\mathrm{NBL}^{+} 10, \mathrm{BAS}^{+} 11\right]$. Finally, and as already sated in the introduction, it is worth underlying that the elastic mismatch between austenite and $\varepsilon$-martensite could generate non-negligible incompatility stresses and image forces on dislocations.

\section{Acknowledgements}

This work was supported by the French State through the program "Investment in the future" operated by the National Research Agency (ANR) and referenced by ANR-11-LABX-0008-01 (LabEx DAMAS).

\section{References}

[BAS $\left.{ }^{+} 11\right]$ O. Bouaziz, S. Allain, C.P. Scott, P. Cugy, and D. Barbier. A high manganese austenitic twinning induced plasticity steels: A review of the microstructure properties relationships. Current Opinion in Solid State and Materials Science, 15:141-168, 2011.

$\left[\mathrm{BBP}^{+} 14\right] \quad$ D. Bouscaud, S. Berveiller, R. Pesci, E. Patoor, and A. Morawiec. Local stress analysis in an sma during stress-induced martensitic transformation by kossel microdiffraction. Advanced Materials Research, 996:45-51, 2014.

[BGE97] N. Bergeon, G. Guenin, and C. Esnouf. Microstructural analysis of the stress-induced $\varepsilon$ martensite in a femnsicrni shape memory alloy: Part icalculated description of the microstructure. Materials Science and Engineering A, 238:309-316, 1997.

[BJS67] D. Berlincourt, H. Jaffe, and L.R. Shiozawa. Electroelastic properties of the sulfides, selenides, and tellurides of zinc and cadmium. Physical Review, 129:1009-1017, 1967.

[CRCS19] X. Chen, T. Richeton, C.Motz, and S.Berbenni. Elastic fields due to dislocations in anisotropic bi- and tri-materials: applications 
to discrete dislocation pile-ups at grain boundaries. International Journal of Solids and Structures, 164:141-156, 2019.

[DDR $\left.{ }^{+} 12\right]$ S. Dancette, L. Delannay, K. Renard, M.A. Melchior, and P.J. Jacques. Crystal plasticity modeling of texture development and hardening in TWIP steels. Acta Materialia, 60:2135-2145, 2012.

[FD67] E.S. Fisher and D. Dever. Temperature dependence of elastic moduli of ruthenium, rhenium, cobalt, dysprosium, and erbium a study of the elastic anisotropy-phase transformation relationship. Transactions of the Metallurgical Society of AIME, 239:48-57, 1967.

[FW74] E.R. Fuller and W.F. Weston. Relation between elastic-constant tensors of hexagonal and cubic structures. Journal of Applied Physics, 45:3772-3776, 1974.

[GMK $\left.{ }^{+} 11\right]$ T. Gebhardt, D. Music, D. Kossmann, M. Ekholm, I.A. Abrikosov, L. Vitos, and J.M. Schneider. Elastic properties of fcc FeMnX (X $=\mathrm{Al}, \mathrm{Si}$ ) alloys studied by theory and experiment. Acta Materialia, 59:3145-3155, 2011.

[GPH05] N. Gey, B. Petit, and M. Humbert. Electron backscattered diffraction study of $\varepsilon / \alpha^{\prime}$ martensitic variants induced by plastic deformation in 304 stainless steel. Metallurgical and Materials Transactions A, 36A:3291-3299, 2005.

[GPK89] J. Gemperlova, V. Paidar, and F. Kroupa. Compatibility stresses in deformed bicrystals. Journal of Physics B, 39:427-446, 1989.

[IRV ${ }^{+}$09] H. Idrissi, L. Ryelandt, M. Veron, D. Schryversa, and P.J. Jacques. Is there a relationship between the stacking fault character and the activated mode of plasticity of femn-based austenitic steels? Scripta Materialia, 60:941944, 2009.

[JD18] P.-A. Juan and R. Dingreville. Elastic greens function in anisotropic bimaterials considering interfacial elasticity. Journal of Elasticity, 2018:277296, 2018.

[KHRN18] W. Kaita, K. Hagihara, L.A. Rocha, and T. Nakano. Plastic deformation mechanisms of biomedical cocrmo alloy single crystals with hexagonal close-packed structure. Scripta Materialia, 142:111-115, 2018. 
[KMRM94] R.R. Kelle, H.J. Maier, H. Renner, and H. Mughrabi. Local internal stress characterization in a tensile-deformed copper single crystal by convergent-beam electron diffraction. Philosophical Magazine A, 70:329-340, 1994.

$\left[\mathrm{LBG}^{+} 18\right]$ S. Lhadi, S. Berbenni, N. Gey, T. Richeton, and L. Germain. Micromechanical modeling of the effect of elastic and plastic anisotropies on the mechanical behavior of $\beta$-ti alloys. International Journal of Plasticity, 109:88-107, 2018.

[LW70] H.J. Leamy and H. Warlimont. The elastic behaviour of ni-co alloys. Physica Status Solidi a, 37:523-534, 1970.

[Mar72] R.M. Martin. Relation between elastic tensors of wurtzite and zinc-blende structure materials. Physical Review B, 6:4546-4553, 1972.

$\left[\mathrm{NBL}^{+} 10\right]$ F.C. Nascimento, J.C. Bueno, C.M. Lepienski, J. Otubo, and P.R. Mei. Determination of the mechanical properties of epsilon martensite by nanoindentation in shape memory stainless alloys. Rem: Revista Escola de Minas, 63:39-44, 2010.

[Nis78] Z. Nishiyama. MartensiticTransformation. AcademicPress,NewYork, 1978.

[PK94] L. Priester and O. Khalfallah. Image force on a lattice dislocation due to a grain boundary in anisotropic f.c.c. materials. Philosophical Magazine, 69:471-484, 1994.

$\left[\mathrm{PNM}^{+} 13\right]$ D.T. Pierce, K. Nowag, A. Montagne, J.A. Jimnez, J.E. Wittig, and R. Ghisleni. Single crystal elastic constants of high-manganese transformation-and twinning-induced plasticity steels determined by a new method utilizing nanoindentation. Materials Science and Engineering A, 578:134-139, 2013.

[RB13] T. Richeton and S. Berbenni. Effects of heterogeneous elasticity coupled to plasticity on stresses and lattice rotations in bicrystals: a Field Dislocation Mechanics viewpoint. European Journal of Mechanics A/Solids, 37:231-247, 2013.

$\left[\mathrm{REH}^{+} 10\right] \quad$ F. Roters, P. Eisenlohr, L. Hantcherli, D. D. Tjahjanto, T. R. Bieler, and D. Raabe. Overview of constitutive laws, kinematics, ho- 
mogenization and multiscale methods in crystal plasticity finiteelement modeling: Theory, experiments, applications. Acta Materialia, 58:1152-1211, 2010.

[Ric17] T. Richeton. Incompatibility stresses aand lattice rotations due to grain boundary sliding in heterogeneous anisotropic elasticity. Crystals, 7:203 (1-14), 2017.

[Rob68] F.N.H. Robinson. Relations between the components of the nonlinear polarisability tensor in cubic and hexagonal iivi compounds. Physics Letters A, 26:435-436, 1968.

[RTBB15] T. Richeton, I. Tiba, S. Berbenni, and O. Bouaziz. Analytical expressions of incompatibility stresses at $\Sigma 3(111)$ twin boundaries and consequences on single-slip promotion parallel to twin plane. Philosophical Magazine, 95:12-31, 2015.

[Sau06] M. Sauzay. Effet de lánisotropie élastique cristalline sur la distribution des facteurs de schmid la surface des polycristaux. Comptes Rendus Mécanique, 334:353-361, 2006.

[SS67] M.L. Shepard and J.F. Smith. Single crystalline elastic constants of lead-thallium alloys. Acta Metallurgica, 15:357-363, 1967.

[ST74] G. Stone and G. Thomas. Deformation induced alpha and epsilon martensites in fe-ni-cr single crystals. Metallurgical Transactions, 5:2095-2102, 1974.

[TRM ${ }^{+}$15] I. Tiba, T. Richeton, C. Motz, H. Vehoff, and S. Berbenni. Incompatibility stresses at grain boundaries in ni bicrystalline micropillars analyzed by an anisotropic model and slip activity. Acta Materialia, 83:227-238, 2015.

[Voi28] W. Voigt. Lehrbuch Der Kristallphysik. B.G. Teubner, Leipzig, Germany, 1928.

[WG75] W.F. Weston and A.V. Granato. Cubic and hexagonal singlecrystal elastic constants of a cobalt-nickel alloy. Physical Review $B, 12: 5355-5362,1975$.

[WMD06] A.J. Wilkinson, G. Meaden, and D.J. Dingley. High-resolution elastic strain measurement from electron backscatter diffraction 
patterns: New levels of sensitity. Ultramicroscopy, 106:306-313, 2006.

[YW92] J.H. Yang and C.M. Wayman. Self-accommodation and shape memory mechanism of $\varepsilon$-martensite - i. experimental observations. Materials Characterization, 28:23-35, 1992.

[Zar63] A. Zarembovitch. Etude des constantes élastiques de la blende et de leur variation avec la température. Le Journal de Physique, 24:1097-1102, 1963. 